\title{
Evidencias pedagógicas de gamificación: autoconstrucción y etnoculturalidad de aprendizajes matemáticos
}

\section{Pedagogical evidence of gamification: self-construction and ethnocultural learning of mathematics}

\author{
Jhon Holguin-Alvarez ${ }^{1}$, Gloria María Villa Córdova ${ }^{2}$, Lizbeth Maricielo Tafur Medrano ${ }^{3}$ y \\ Yasmín Iveth Chávez Álvarez ${ }^{4}$
}

Universidad César Vallejo, Lima, Perú ${ }^{1234}$

Orcid ID: https://orcid.org/0000-0001-5786-0763 ${ }^{1}$

Orcid ID: https://orcid.org/0000-0003-3038-94432

Orcid ID: https://orcid.org/0000-0001-7730-6508 3

Orcid ID: https://orcid.org/0000-0002-4097-0621

Recibido: 12 de noviembre 2018

Aceptado: 01 de julio 2019

\section{Resumen}

Los estudios de gamificación en educación se han incrementado debido a la dificultad del profesorado para abordar aprendizajes rígidos. En ese sentido, las matemáticas son complejas para la didáctica en escolaridad. Por ello, en el presente estudio se describen dos estudios de corte cuantitativo, con diseño experimental, con el propósito de determinar los efectos de dos tipos de gamificación: a) autoconstructiva y b) etnocultural, a partir de las variables "aprendizaje de la adición y sustracción" y "resolución de problemas" respectivamente. Se incluyeron 80 estudiantes de cuarto grado de primaria en el experimento A ( $M=10.3$ años; D.E. $=1.54)$, y en el experimento $B$ se indujo a 36 estudiantes de primer grado $(M=6.4$ años; D.E.=1.36). Para esta investigación se utilizaron dos instrumentos: Prueba de resolución de problemas y Prueba de medición para el aprendizaje de la adición y sustracción. Del experimento A, se concluye que las técnicas etnoculturales desarrollaron la resolución de problemas con efectos significativos en el grupo experimental; los sujetos de dicho experimento desarrollaron problemas de comparación e igualación con mejores indicadores estadísticos. Por su parte, el experimento B concluye que los efectos fueron positivos en el aprendizaje de operaciones de adición y sustracción, con más beneficios para la representación algorítmica y simbólica.

\footnotetext{
${ }^{1}$ Correspondencia al autor

E-mail: jhonholguinalvarez@gmail.com
} 
Palabras claves: gamificación; aprendizaje autónomo; técnica etnocultural; resolución de problemas; operaciones matemáticas.

\begin{abstract}
The studies of gamification in education have increased due to the difficulty of the teaching staff to tackle rigid learning. In this sense, mathematics is complex for didactics in schooling. Therefore, this research describes two quantitative studies, with experimental design. The purpose was to determine the effects of two types of gamification: a) selfconstructive and b) ethnocultural, in the variables learning of addition and subtraction and problem solving respectively. We included 80 fourth grade students in experiment $\mathrm{A}(\mathrm{M}=10.3$ years old, $\mathrm{SD}=$ 1.54), and in experiment $B$ we induced 36 first grade students $(M=6.4$ years old, $S D=1.36)$. We used two instruments: Problem Solving Test and Measurement Test for Learning Addition and Subtraction. From experiment A, we concluded that, ethnocultural techniques developed the resolution of problems with significant effects in the experimental group, the subjects of this experiment developed problems of comparison and equalization with better statistical indicators. Experiment B concluded that the effects were positive in the learning of addition and subtraction operations; with more benefits for the algorithmic and symbolic representation.
\end{abstract}

Keywords: gamification; autonomous learning; ethnocultural technique; problem resolution; mathematical operations.

\title{
Introducción
}

La gamificación en el aula se ha convertido en una tendencia actual en distintos contextos educativos, ya que su naturaleza plasma un atractivo único, sobre todo para grupos estudiantiles con dificultades para desarrollar aprendizajes rígidos; es decir, aquellos aprendizajes complejos que se encuentran determinados por la maduración del sujeto, los procesos cognitivos y por otros factores como la motivación. El desarrollo de habilidades cognitivas desde la interacción lúdica empodera los procesos autónomos del aprendizaje desde distintas fuentes de información procesadas en la práctica constante de la matemática. Algunas capacidades matemáticas necesitan el desarrollo de procesos cognitivos superiores e inferiores del aprendizaje, los cuales acompañan determinados procesos poco interactivos (Calsa \& Furtuoso, 2015; Tafarelo \& Bonano, 2016; Tadia e D’Amore, 2015), y otros mucho más interactivos implican el desarrollo de análisis y síntesis, sobre todo entre los 6 y 10 años de edad, etapa en la cual el estudiante necesita del apoyo condicional para el desarrollo de mayor interactividad en el pensamiento matemático.

\section{Evidencias en gamificación auto constructiva del aprendizaje del cálculo}

La experiencia de gamificación produce mejores situaciones cognitivas en el sujeto, el placer por la realización de la actividad interviene en la ejecución de capacidades (Carlson, Harris \& Harris, 2017), como por ejemplo el razonamiento, por lo que se aligera la memoria 
operativa y se produce el refuerzo de determinadas metas académicas. En el intercambio de información matemática se establecen puentes entre los procesos de operatividad lógica y las habilidades matemáticas (Bragg \& Herbert, 2017; Çelik, 2017; Wong, 2018). Este proceso placentero que provoca el juego en la operatividad, modifica las estructuras cognitivas (Feurstein en Alpízar, 2016), ya que el juego posee mecanismos de feedback correctivo ante la probabilidad del error y asegura la capacidad de supervivencia/latencia del procesamiento cognitivo de las operaciones durante espacios de tiempo más prolongados, mejora la atención y disminuye la recarga de la memoria operativa (Groos, 1976; Gross, en Martínez, 2008).

El intercambio de información en cantidades se realiza en el proceso de aprendizaje de operaciones de cálculo, ya que el sujeto necesita desarrollar procesos de iconicidad, simbolización y algoritmización (Carrillo et al., 2016; Cañellas y Rassetto, 2013; Mañeru, 2013). En el primer proceso, el sujeto adquiere los esquemas espaciales del contenido numérico para su representación, en la segunda, el sujeto adiciona una cifra o valor numérico a las cantidades representadas, con el fin de utilizar pasos específicos en la transformación de cantidades en el proceso de representación algorítmica.

El proceso de aprendizaje del cálculo también implica la habilidad para resolver problemas, por lo que son procesos coadyuvantes entre sí, en dicha resolución el estudiante necesita sentir mayor afecto hacia las tareas cognitivas complejas con el fin de superarlas (Ayal et al., 2015; Lubis \& Nasution, 2017; Ortiz-Colón, Jordán \& Agredal, 2018). En este caso, el proceso de gamificación estimula el proceso de construcción de información desde la concatenación de otras fuentes información. En otras palabras, los sujetos que resuelven problemas aplican múltiples procesos cognitivos, de cuya ejecución obtienen información nueva mediante las distintas operaciones matemáticas.

En el caso de la capacidad de operaciones matemáticas, la gamificación ha demostrado que la interacción pedagógica mediante el juego permite el desarrollo de variables como el conteo matemático (Meloni et al., 2017; Yorulmaz \& Onal, 2017), las capacidades de clasificación y numeración (Cánovas, 2016; Siegler \& Braithwaite, 2016; Schneider, 2015; Sungwoong, 2016). Por lo tanto, la gamificación como estrategia pedagógica permite el logro de habilidades cognitivas en dos de sus competencias matemáticas más complejas de desarrollar en el aula como lo es el aprendizaje del cálculo numérico y la resolución de problemas, ya que en ellas intervienen múltiples procesos de desarrollo. 


\section{Evidencias en técnicas etnoculturales para el aprendizaje de resolución de problemas}

La presencia de asignaciones etnoculturales en las transacciones pedagógicas, han permitido el logro de procesos de resolución de problemas como una modalidad de enseñanza lúdica (Lee, 2016; Lozano, 2017; Viterbori, Traverso \& Usai, 2017), en algunos casos, se asume que la responsabilidad del aprendizaje recae únicamente en la acción pedagógica, sin tomar en cuenta la culturización, ya que es un elemento primordial de enseñanza, influencia directa en la construcción de los aprendizajes de determinados grupos escolares, por su calidad de uso, colores, rasgos ancestrales y significados culturales a los que se enfrentan en su proceso educativo.

La resolución de problemas es una capacidad flexible, por dicha naturaleza se pueden formular esquemas erróneos en los escolares, con sistemas ajustados a la lógica, pero que dejan de lado la creatividad analítica (D’Amore e Fandiño, 2013; D’Amore e Fandiño, 2006; D’Amore, 2006; D’Amore; Fandiño e Marazzani, 2004); si se persiste en retroalimentar esquemas erróneos de solución, entonces el concepto de solución puede también ser erróneo y como conocimiento puede perdurar hasta los estudios universitarios ( $\mathrm{D}^{\prime}$ Amore e Fandiño, 2013). Por ejemplo, la búsqueda de soluciones esquematizadas por el modelo: datos, operación, respuesta y comprobación, lo cual actualmente se ha convertido en un sistema vertical de aprendizaje de solución de problemas muy aplicado por docentes en el Perú. Es preocupación de la didáctica de la matemática, y sobre todo desde un enfoque lúdico, crear nuevos puentes entre la pedagogía flexible hacia la complejidad matemática, con el fin de desarrollar el pensamiento de resolución de problemas como una competencia no rígida en el estudiante. La resolución de problemas conceptualmente es comprendida como el proceso de utilización de saberes previos (Flores y Rico, 2015; Segovia y Rico, 2015), aplicados a procesos matemáticos ergonómicos a una situación problemática determinada, ya sea lingüística o numérica. En dicho proceso, el sujeto establece modalidades de solución pertinentes a una situación planteada con el fin de darle objetividad, orden y fin a dicha modalidad de solución. Este proceso suele ser rígido cuando el sujeto que resuelve busca situaciones directas, verticales, atenuados por la rapidez, y sin utilizar estrategias de soporte cognitivo, como, por ejemplo, el intercambio de información, la interacción de su comprensión y el co-análisis de situaciones predictivas.

La gamificación basada en etnoculturalidad ha demostrado que la internalización de procesos de resolución desde el uso de elementos culturales predispone las estructuras cuando se aplica la imaginación sensorial y creativa (Wibowo et al., 2017), en algunos casos el material autóctono proporciona libertad al estudiante, sobretodo porque permite alejarlo del desarrollo de esquemas de solución tradicional, para utilizar la lenguaje como aspecto sociocultural 
primordial en el desarrollo de problemas en equipo (Planas, 2018; Ru-Fen, 2016), y en otro casos, se ha encontrado que los materiales didácticas estructurados como el Tangram, multibase y regletas son más efectivos si se asemejan más al origen cultural de los sujetos que los manipulan (Murillo, Román y Atrio, 2016).

Por todo lo descrito, es propósito del presente estudio determinar los efectos de dos experimentaciones pedagógicas basadas en A) técnicas etnoculturales para la resolución de problemas aditivos y B) gamificación autoconstructiva en el aprendizaje del cálculo de operaciones, en el nivel primaria de Educación Básica Regular del Perú.

\section{Método}

La investigación se desarrolló en el diseño experimental, de corte cuantitativo. En cuanto a la experimentación A (técnicas etnoculturales), se ha seguido el tipo de diseño cuasi experimental con dos grupos de medición (grupo control y experimental), y en razón del experimento B (gamificación autoconstructiva), se ha desarrollado en el tipo de diseño preexperimental con grupo único de experimentación.

Experimentación A: técnicas etnoculturales.

\section{Sujetos}

La muestra fue no probabilística, y se compuso por 80 estudiantes del cuarto grado de primaria de una institución educativa de Lima. Los estudiantes conformaron dos subgrupos con el fin de obedecer a un diseño cuasi experimental (grupo experimental (técnicas etnoculturales) $=40$ sujetos; grupo control (sin tratamiento) $=40$ sujetos). El rango de edad de los participantes fue de entre 10 y 11 años $(M=10.3$; D.E.= 1.54). En cuanto al género la muestra se distribuyó con cierta equidad (varones: $56 \%$; mujeres: $44 \%$ ). Todos eran estudiantes de estrato socio económico bajo. Cabe señalar que se aplicaron criterios de exclusión a sujetos con retraso mental moderado, y niños con constantes problemas de conducta en los últimos dos meses anticipados a la investigación. Estos sujetos participaron en el programa experimental, pero sus datos respecto a la variable se obviaron con el fin de obedecer a dichos criterios. Finalmente, todos participaron mediante la aceptación del consentimiento informado realizado con dirección de la institución y los padres de familia.

\section{Materiales y procedimiento.}

La Prueba de resolución de problemas - PRPM (ad hoc) es una prueba que consta de 16 ítems de respuesta abierta y posibilidad de codificación dicotómica o politómica. Para la 
investigación se utilizaron tres puntuaciones en la resolución de problemas: logro $=2$ puntos; proceso $=1$ puntos; inicio $=0$ puntos. Se desarrolla en aproximadamente en una hora, y evalúa la resolución de problemas de tipo cambio, combinación, comparación e igualación. Esta se sometió a criterio de jueces, en cuyo proceso participaron 5 jurados evaluadores del contenido del instrumento, se obtuvo la aceptación de $100 \%$ como resultado. En cuanto a la fiabilidad se desarrolló el plan de pilotaje con 30 estudiantes del cuarto grado de primaria de instituciones educativas del distrito de Comas, Lima. Como evidencia de confiabilidad, se obtuvo un índice Alfa de Cronbach de 0.83 del total de ítems, por lo que se consideró un instrumento confiable para su aplicación en los grupos del experimento.

En cuanto al procedimiento de experimentación, se acordó con la institución educativa la participación de los estudiantes por cada nómina escolar, se procedió al ajuste de 40 actividades de aprendizaje basadas en técnicas etnoculturales. Estas debían homologarse al plan curricular de la institución educativa con el fin de minimizar la evidencia del desarrollo del plan experimental por parte de los estudiantes, ya que sería una fuente de invalidación interna debido a la predisposición o la negatividad de ellos hacia el programa. Las 40 actividades se basaron en el enfoque cultural vigotskiano, desarrolladas en cuatro mecanismos de acción establecidos por semanas (figura 1): a) conocimiento del cálculo incaico, b) adaptación a los materiales del entorno, c) construcción del conocimiento en operaciones básicas, y d) uso de estrategias. El total de actividades incluyeron 20 recursos alimenticios (habas, maíz, huevos, frejoles, chicha de jora, entre otros), como también materiales de alcance didáctico.

A

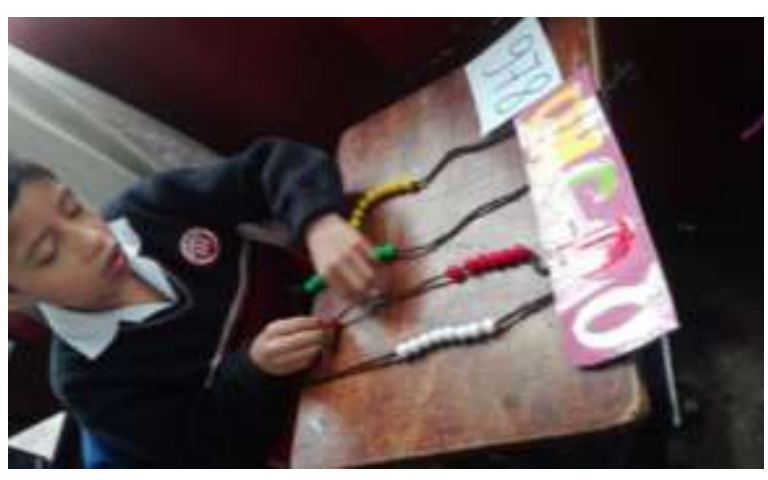

B
D

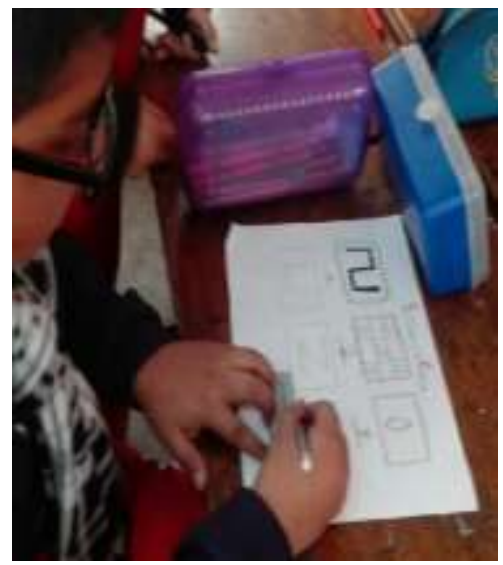

E 


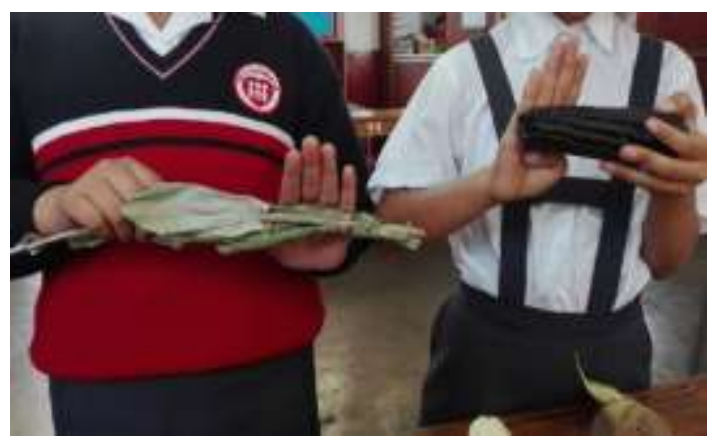

$\mathbf{C}$

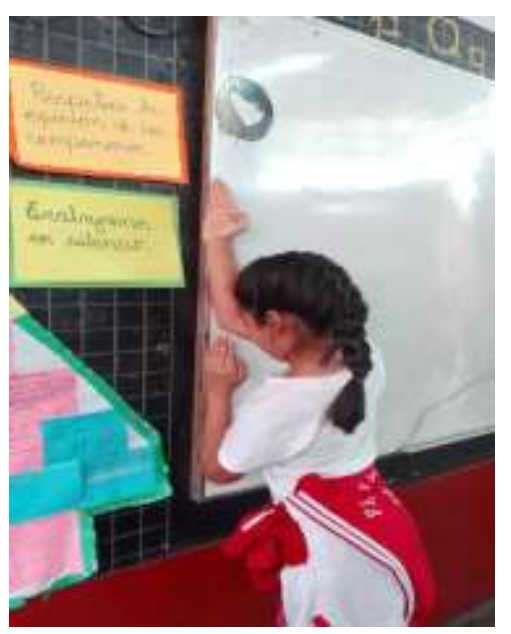

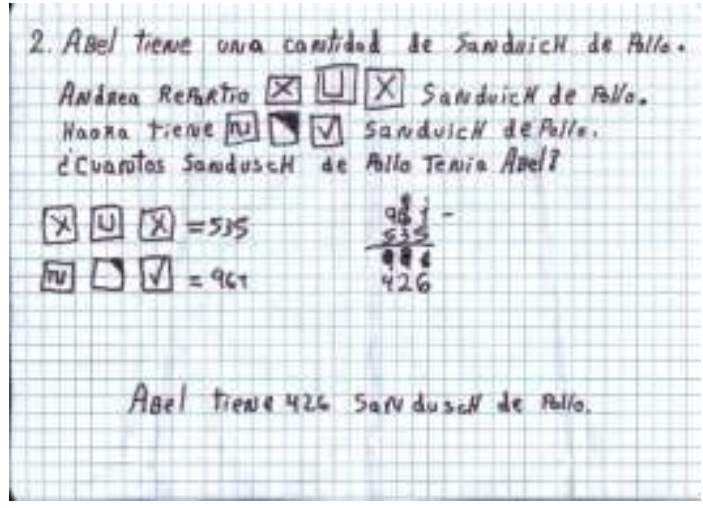

$\mathbf{F}$

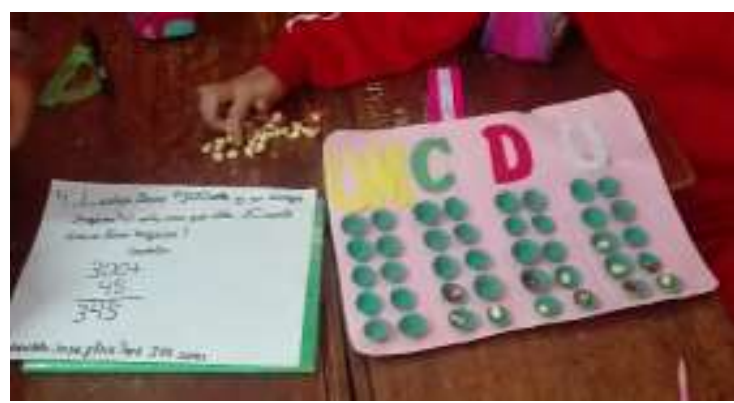

Figura 1. Desarrollo de los mecanismos de acción del programa de técnicas etnoculturales en el grupo experimental de acuerdo a los recursos y materiales implementados.

Fuente: Recursos de la investigación.

Nota: A y $\mathrm{B}=$ mecanismo conocimiento del cálculo incaico (actividades: $\mathrm{A}=$ conoce el Quipu; $\mathrm{B}=$ medición capa); $\mathrm{C}=$ mecanismo adaptación a los materiales $(\mathrm{C}=$ medición Cuchuch $)$; $\mathrm{D}$ y E = mecanismo construcción del conocimiento en operaciones básicas (actividades: $\mathrm{D}=$ medición de allpa; $\mathrm{E}=$ desarrollo mis operaciones); $\mathrm{F}=$ mecanismo uso de estrategias (actividad: $\mathrm{F}=$ utilizo la Yupana).

El desarrollo del programa se complementó en cuatro meses. Una vez recabados los datos del grupo experimental y control, se comprobó su distribución estadística y si estos se ajustaban a la normal, por lo que se comprobó que los datos recogidos sobre la variable resolución de problemas no se ajustaban a la distribución de normalidad $\left(\mathrm{K}-\mathrm{S}_{\text {(pretest) }}=, 116\right.$; K$\left.\mathrm{S}_{\text {(postest) }}=, 112 ; \mathrm{p}<.01\right)$, y en las dimensiones las significancias también fueron idénticas (sig. $=, 000 ; \mathrm{p}<.01)$, por cuanto los datos debían analizarse con pruebas estadísticas no paramétricas para muestras independientes (U de Mann Whitney), en el resultado de contraste general como también en el especifico, sobre todo, si se contaban con datos de categoría ordinal (logro, proceso, inicio). 


\section{Resultados}

Tabla 1

Promedio y desviación estándar en medición pretest y postest de la resolución de problemas y las dimensiones tipo cambio, combinación, comparación e igualación entre los grupos control y experimental (técnicas etnoculturales)

\begin{tabular}{lccccc}
\hline \multirow{2}{*}{ Grupos y mediciones } & \multirow{2}{*}{ RP } & \multicolumn{4}{c}{ *Resolución tipo... } \\
\cline { 3 - 6 } & & cambio & combinación & comparación & igualación \\
\hline Grupo control-pretest & $4.38(2.0)$ & $0.975(1.0)$ & $1.05(0.9)$ & $1.25(1.1)$ & $1.1 .(1.0)$ \\
Grupo experimental-pretest & $4.1(1.4)$ & $1.075(1.0)$ & $1.05(1.0)$ & $1(0.8)$ & $0.975(0.9)$ \\
Grupo control-postest & $4.1(1.4)$ & $1.075(1.0)$ & $1.05(1.0)$ & $1(0.8)$ & $0.975(0.9)$ \\
Grupo experimental-postest & $12.03(3.2)$ & $3.08(1.2)$ & $3.23(1.3)$ & $2.85(1.4)$ & $2.88(1.4)$ \\
\hline Fuente:
\end{tabular}

Fuente: Base de datos de la investigación.

Nota: *promedio (desviación estándar); RP = resolución de problemas.

Tabla 2

Comparación pretest y postest en la aplicación del programa de técnicas etnoculturales en la resolución de problemas

\begin{tabular}{ccccc}
\hline Mediciones & Grupos & Rp & Sr & U \\
\hline \multirow{2}{*}{ Pretest } & Control & 42,25 & 1690,00 & 730,000 \\
& Experimental & 38,75 & 1550,00 & \\
\hline \multirow{2}{*}{ Postest } & Control & 22,73 & 909,00 & \multirow{2}{*}{$89,000^{*}$} \\
& Experimental & 58,28 & 2331,00 & \\
\hline
\end{tabular}

Fuente: Base de datos de la investigación.

Nota: $* \mathrm{p}<.01 ; \mathrm{Rp}=$ Rango promedio; $\mathrm{Sr}=$ Suma de rangos; $\mathrm{U}=$ índice de Mann Whitney.
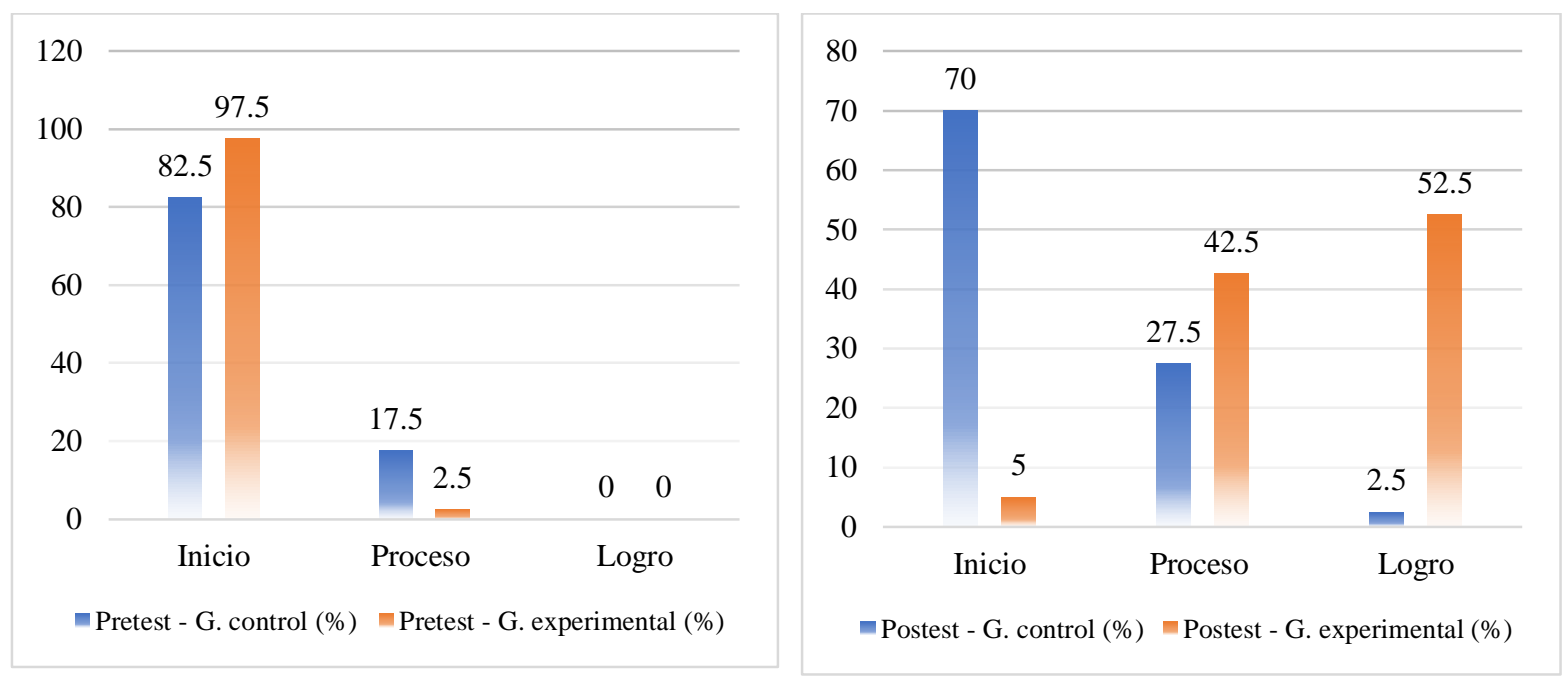

Figura 2. Porcentajes de medición pretest y postest en la resolución de problemas de acuerdo a los niveles inicio, proceso y logro de los grupos control y experimental (técnicas etnoculturales).

Fuente: Base de datos de la investigación. 
Tabla 3

Comparación de las mediciones pretest y postest en la aplicación del programa de técnicas etnoculturales en la resolución de problemas de tipo cambio, combinación, comparación e igualación

\begin{tabular}{|c|c|c|c|c|c|}
\hline Tipo de RP & Mediciones & Grupos & $\mathrm{Rp}$ & $\mathrm{Sr}$ & $\mathrm{U}$ \\
\hline \multirow{4}{*}{ Cambio } & \multirow{2}{*}{ Pretest } & Control & 39,04 & 1561,50 & \multirow{2}{*}{741,500} \\
\hline & & Experimental & 41,96 & 1678,50 & \\
\hline & \multirow{2}{*}{ Postest } & Control & 26,26 & 1050,50 & \multirow{2}{*}{230,500} \\
\hline & & Experimental & 54,74 & 2189,50 & \\
\hline \multirow{4}{*}{ Combinación } & \multirow{2}{*}{ Pretest } & Control & 40,55 & 1622,00 & \multirow{2}{*}{798,000} \\
\hline & & Experimental & 40,45 & 1618,00 & \\
\hline & \multirow{2}{*}{ Postest } & Control & 26,06 & 1042,50 & \multirow{2}{*}{222,500} \\
\hline & & Experimental & 54,94 & 2197,50 & \\
\hline \multirow{4}{*}{ Comparación } & \multirow{2}{*}{ Pretest } & Control & 42,15 & 1686,00 & \multirow{2}{*}{734,000} \\
\hline & & Experimental & 38,85 & 1554,00 & \\
\hline & \multirow{2}{*}{ Postest } & Control & 29,44 & 1177,50 & \multirow{2}{*}{357,500} \\
\hline & & Experimental & 51,56 & 2062,50 & \\
\hline \multirow{4}{*}{ Igualación } & \multirow{2}{*}{ Pretest } & Control & 41,73 & 1669,00 & \multirow{2}{*}{751,000} \\
\hline & & Experimental & 39,28 & 1571,00 & \\
\hline & \multirow{2}{*}{ Postest } & Control & 28,91 & 1156,50 & \multirow{2}{*}{336,500} \\
\hline & & Experimental & 52,09 & 2083,50 & \\
\hline
\end{tabular}

Fuente: Base de datos de la investigación.

Nota: ${ }^{*} \mathrm{p}<.01 ; \mathrm{RP}=$ Resolución de problemas; $\mathrm{Rp}=$ Rango promedio; $\mathrm{Sr}=$ Suma de rangos; $\mathrm{U}=$ índice de Mann Whitney.

Experimentación B: gamificación autoconstructiva.

\section{Sujetos}

La muestra de esta experimentación fue de tipo no probabilística, de única experimentación en la modalidad de diseño pre experimental, con mediciones pretest y postest. El total de participantes fue de 36 estudiantes del primer grado de una institución educativa de Lima. La distribución por género fue equitativa (varones: $50 \%$; mujeres: $50 \%$ ), la edad se encontró entre el rango de 6 a 7 años de edad (M=6.4; D.E.=1.36). Ninguno de los estudiantes implicados en el estudio presentaron diferencias a nivel cognitivo, afectivo y conductual, por lo que se consideró la participación de todos los sujetos en el programa de gamificación autoconstructiva. Todos los sujetos del estudio fueron considerados como participantes del estudio luego de que sus padres aceptasen el trabajo con sus hijos. Este proceso se realizó mediante la gestión del consentimiento informado. 


\section{Materiales y procedimiento}

La Prueba de medición para el aprendizaje de la adición y sustracción - PMAAS (ad hoc) es un instrumento de tipo cuantitativo, construido con un total de 12 ítems con medición de aciertos ( 1 punto), y errores ( 0 puntos) en la respuesta de cada ítem, se resuelve entre 45 y 60 minutos. El PMAAS mide el aprendizaje de operaciones de adición y sustracción en tres procesos cognitivos: representación icónica, simbólica y algorítmica; y fue sometido a evaluación mediante el procedimiento de juicio de expertos, en cuyo proceso participaron 5 evaluadores especialistas en didáctica de la matemática, evaluación y aprendizajes, como también metodólogos de investigación. La aprobación en validez de contenido fue mayor a 97 $\%$ del total de ítems. Una vez validada la prueba de evaluación, se procedió a la realización de un plan piloto con 20 estudiantes de primer grado de primaria de instituciones educativas de Lima, tras el cálculo de confiabilidad Kuder-Richardson, se obtuvo un índice de consistencia de 0.91 , por lo que la estructura general del dicho instrumento fue aceptable para el estudio.

El procedimiento de experimentación se realizó luego de elaborar 45 actividades pedagógicas basadas en el enfoque de autoconstrucción de los aprendizajes lúdicos, cuya naturaleza teórica provenía de las propuestas de Groos sobre el juego instruccional. En la elaboración del programa se consideraron utilizar un total de 154 recursos y materiales relacionados al entorno de los participantes (chapas, latas, hojas de papel, plumones, lapiceros; etcétera), los cuales sirvieron para recrear distintos momentos del programa de gamificación autoconstructiva con los participantes.

G

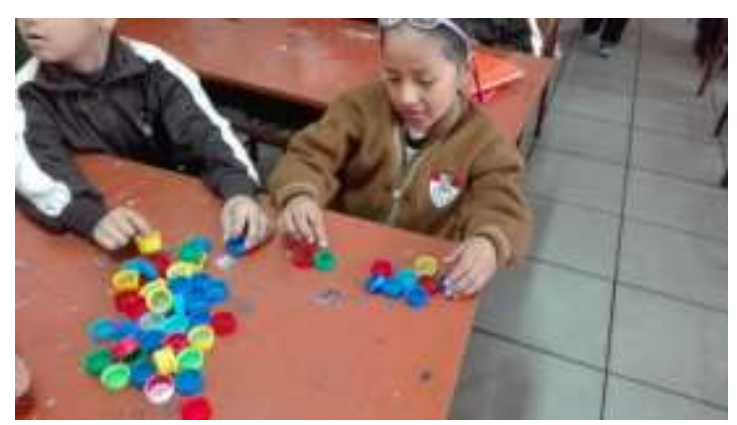

$\mathbf{H}$

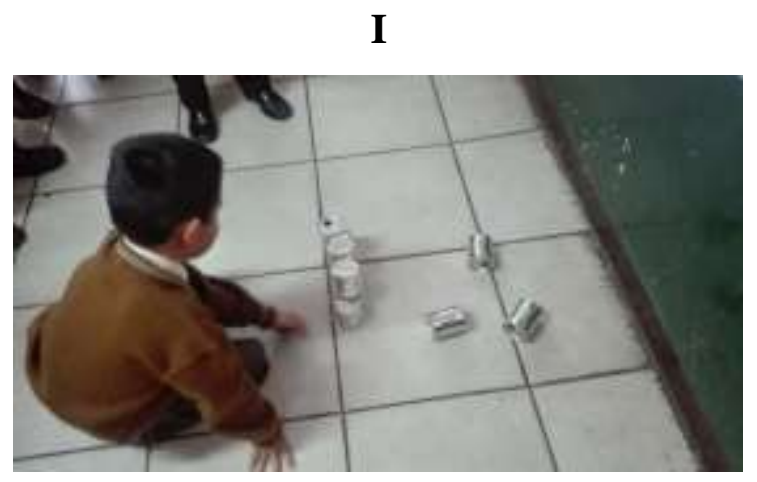

$\mathbf{J}$ 

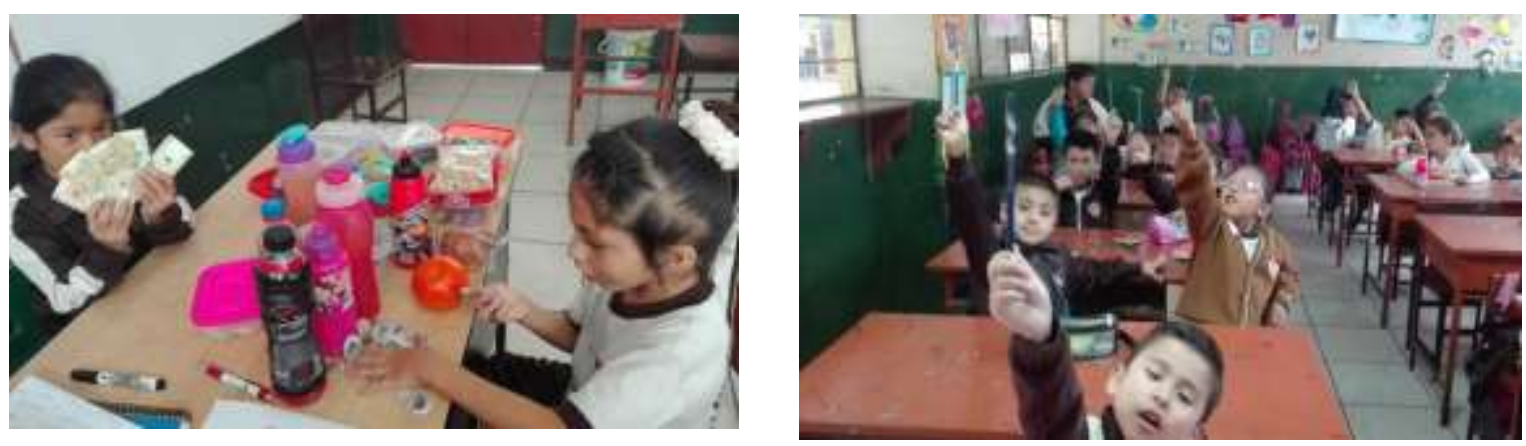

Figura 3. Desarrollo de los mecanismos de acción del programa de gamificación autoconstructiva en el grupo de experimentación.

Fuente: Recursos de la investigación.

Nota: $\mathrm{G}$ = fase de distribución, actividades chapitas de colores; $\mathrm{H}$ = Fase de ejecución, la tiendita; $\mathrm{I}$ = fase de ejecución, actividad tumbalatas; $\mathrm{J}=$ fase de logro, actividad los lapiceros viajan.

Las fases del programa se plantearon de acuerdo a tres mecanismos de trabajo desarrollados en el aula, los cuales fueron denominados como: primera fase = distribución, segunda fase $=$ ejecución; y tercera fase $=$ logro. A su vez, en estas fases se utilizaron los recursos antes mencionados (figura 2). Cabe señalar que estas fases del programa se desarrollaron por igual en las 45 actividades a desarrollar, ya que los sujetos recién se encontraban en etapa de formación escolar (6 a 7 años de edad) y debían seguir pasos muy similares a los de la programación curricular de primer grado; de esa forma se evitaría que percibiesen el cambio entre las distintas etapas del programa. Finalmente, las 45 actividades se desarrollaron en un aproximado de 5 meses con total normalidad. Una vez recogidos los datos de las mediciones pretest y postest, estos se analizaron con el fin de saber si se distribuían con criterio de normalidad. Para esto, se utilizó la prueba Kolmogorov-Smirnov ( $\mathrm{n}>30$ individuos), de cuyo análisis se obtuvieron distribuciones no ajustadas a la normalidad (tabla 4), lo cual sugirió que los resultados se analizaran con la prueba de los signos. De igual modo se comprobó la distribución de normalidad por la prueba Shapiro-Wilk.

\section{Tabla 4}

Distribuciones en los datos y significancias de las pruebas Kolmogorov-Smirnov y ShapiroWilk en el análisis de los datos de variable y dimensiones

\begin{tabular}{|c|c|c|c|c|c|c|c|}
\hline \multirow{2}{*}{ Medición } & \multirow{2}{*}{ Dimensiones o variable } & \multicolumn{3}{|c|}{ Kolmogorov-Smirnov ${ }^{\mathrm{a}}$} & \multicolumn{3}{|c|}{ Shapiro-Wilk } \\
\hline & & Estadístico & $\mathrm{Gl}$ & Sig. & Estadístico & $\mathrm{gl}$ & Sig. \\
\hline \multirow{4}{*}{ Pretest } & Representación icónica & ,295 & 36 &, 000 &, 792 & 36 &, 000 \\
\hline & Representación simbólica & ,245 & 36 & ,000 &, 835 & 36 &, 000 \\
\hline & Representación algorítmica & ,368 & 36 &, 000 &, 722 & 36 &, 000 \\
\hline & Aprendizaje de sustracción y adición &, 212 & 36 &, 000 & ,922 & 36 &, 015 \\
\hline
\end{tabular}




\begin{tabular}{llllllll}
\hline & Representación icónica &, 257 & 36 &, 000 &, 806 & 36 &, 000 \\
\multirow{2}{*}{ Postest } & Representación simbólica &, 279 & 36 &, 000 &, 781 & 36 &, 000 \\
& Representación algorítmica &, 283 & 36 &, 000 &, 769 & 36 &, 000 \\
& Aprendizaje de sustracción y adición &, 203 & 36 &, 001 &, 901 & 36 &, 004 \\
\hline
\end{tabular}

Fuente: Base de datos de la investigación.

Nota: a. Corrección de significación de Lilierfors.; gl = grados de libertad; sig. = significancia o p-valor.

\section{Resultados}

\section{Aprendizaje de la sustracción y adición}

En cuanto a las diferencias entre las medidas pretest y postest, se han encontrado diferencias significativas $(\mathrm{d}(+)=36 ; \mathrm{Z}=-5,833$; sig. $=, 000)$, de igual modo, en las evidencias por cada dimensión (tabla 5); se evidenciaron los beneficios de la gamificación autoconstructiva entre las mediciones pretest y postest, como también en su perspectiva descriptiva (figura 4).

\section{Tabla 5}

Comparación de las mediciones pretest y postest en la representación icónica, simbólica y algorítmica de la adición y sustracción

\begin{tabular}{ccccc}
\hline Representación & Diferencias positivas & Empates & $\mathrm{Z}$ & Sig. \\
\hline Icónica & 34 & 2 & $-5,659$ &, 000 \\
Simbólica & 33 & 3 & $-5,570$ &, 000 \\
Algorítmica & 34 & 2 & $-5,659$ &, 000
\end{tabular}

Fuente: Base de datos de la investigación.

Nota: $\mathrm{Z}=$ Distribución; Sig. = significancia o p-valor.

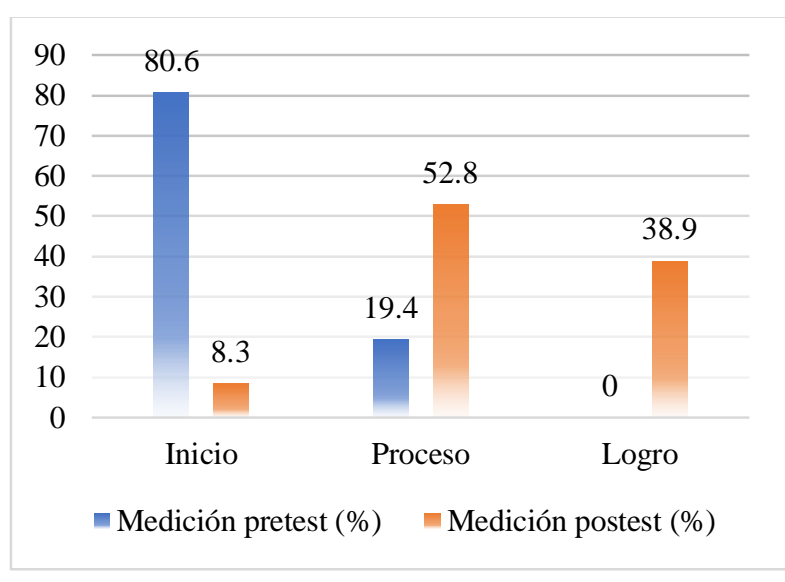

Representación icónica

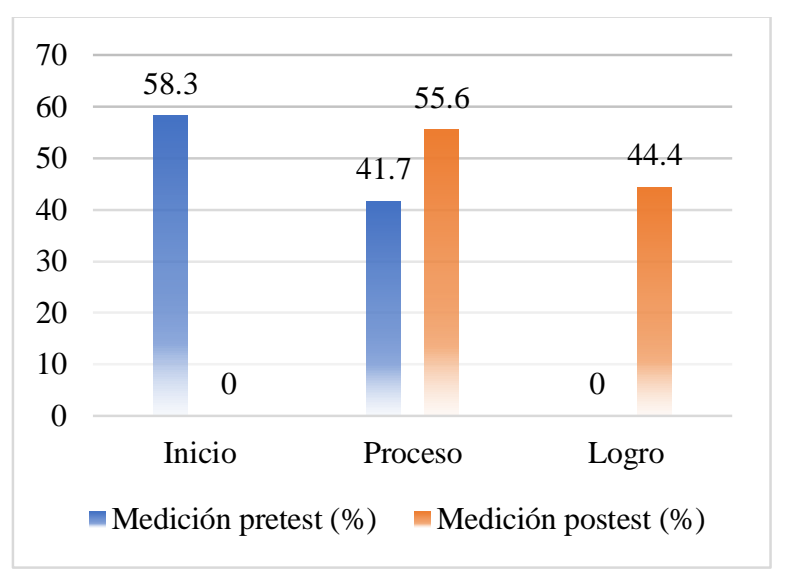

Representación simbólica 


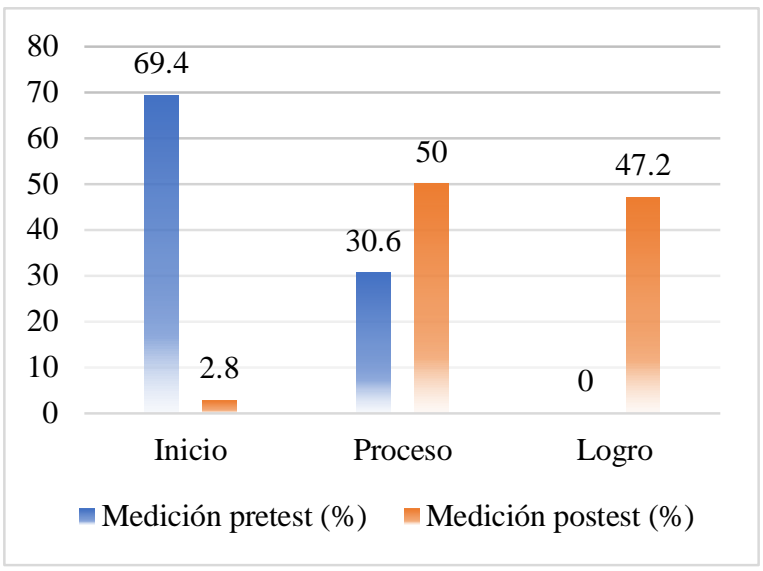

Representación algorítmica

Figura 4. Porcentajes de las mediciones pretest y postest en el aprendizaje de la sustracción y adición en su modalidad representación icónica, simbólica y algorítmica.

Fuente: Base de datos de la investigación.

\section{Discusión}

El primer objetivo de investigación se centró en determinar los efectos de las técnicas etnoculturales en la resolución de problemas de 40 niños y niñas que recibieron el programa de experimentación. Los primeros hallazgos basados en la igualdad encontrada en la variable resolución de problemas antes de ejecutar el programa $(U$ (medición pretest) $=730,000 ; Z=-, 685 ; p$ $>.01$ ), expresó la estabilidad o equilibrio encontrado entre los grupos experimental y control. Sin embargo, luego de la realización del programa se encontraron diferencias que establecieron la efectividad de dichas técnicas $(\mathrm{U}$ (medición postest) $=89,000 ; \mathrm{Z}=-, 6862 ; \mathrm{p}<.01$ ), lo que demostró que las técnicas etnoculturales fueron efectivas en el $50 \%$ de estudiantes que llegaron a nivel de logro en el grupo experimental (figura 2). Estos resultados han sido similares a los encontrados en experiencias sobre el desarrollo de procesos cognitivos relacionados con la resolución de problemas, y de los cuales resaltan los trabajos sobre interactividad con el profesor (Calsa \& Furtuoso, 2015; Tafarelo \& Bonano, 2016). Debido a la recarga cognitiva que implica la resolución de una operación o de un problema, es necesario acompañar el aprendizaje con estrategias de anclaje, es decir, con apoyo del docente en paralelo a los tiempos de resolución que el estudiante realiza en la búsqueda de soluciones, sobretodo, en niños y niñas menores de 10 años de edad como se implementó en el procedimiento del experimento.

El esquema de culturización de la resolución de problemas ha cambiado rotundamente los esquemas de solución que utilizan los estudiantes en el aula: recojo de datos-operaciónrespuesta (D’Amore e Fandiño, 2013; Lozano, 2017; Viterbori et al., 2017). Este tipo de evidencias se relacionan con lo encontrado en este estudio, ya que los rasgos culturales como 
colores, uso de figuras planas como en volumen, uso del espacio, forma y otros extraídos de recursos incaicos como en el programa de técnica etnoculturales (figura 1), han sido determinantes para que los estudiantes desarrollen con menor probabilidad de fracaso los diferentes problemas de evaluación. En cierta medida, estos rasgos etnoculturales han brindado mejor adaptabilidad al problema (Lee, 2016; Viterbori et al., 2017), debido al potencial lúdico que representa desarrollar un problema desde el medio o recurso intercultural, lo que incrementó la posibilidad de aciertos luego del desarrollo del programa. En este caso, la flexibilidad cognitiva ha sido posible por el desarrollo de la capacidad de análisis en los niños del grupo experimental, lo cual confirma la teoría antepuesta sobre resolución de operaciones (D’Amore e Fandiño, 2013; D’Amore e Fandiño, 2006; D’Amore, 2006), y se ha influenciado en el pensamiento del estudiante con el fin de incrementar su imaginación sensorial y creativa para desarrollar soluciones inesperadas o lograr obtener resultados con mecanismos sin verticalidad (Wibowo et al., 2017).

Esta última apreciación se pudo observar en un número considerable de estudiantes del grupo experimental, pues en la resolución de problemas de tipo cambio: el $67.5 \%$ que se ubicó en nivel de inicio, $30 \%$ en nivel de proceso, y $2.5 \%$ en nivel de logro en la medición pretest, disminuyó en el nivel de inicio a $15 \%$, aumentó en el nivel de proceso a $32.5 \%$, y a $52.5 \%$ en el nivel de logro, comparado con los resultados en el grupo control. En cuanto a la resolución de problemas de tipo combinación: el $62.5 \%$ de nivel de inicio se redujo al $15 \%$ en la medición postest, y del 37.5 al $20 \%$ en el nivel de proceso, como también aumentó del 0 al $65 \%$ de estudiantes del grupo experimental ubicados en el nivel de logro. En cuanto a los problemas de comparación: el nivel de proceso aumentó de 30 a $32.5 \%$, y del 0 al $45 \%$ en el nivel de logro.

Finalmente, en la resolución de problemas de igualación, el incremento fue de $50 \%$ en el nivel de logro del grupo experimental. Esto confirma que el programa es útil en otros aspectos desarrollados para cada tipo de resolución de problemas, de lo cual se infiere que la libertad en la resolución de ejercicios que el estudiante desarrolla, es necesaria para que se mejore el uso de otros recursos cognitivos, como el mejor procesamiento a nivel de memoria operativa, y así desestimar el desgaste o recarga cognitiva por cada paso que implica la búsqueda de soluciones; esto también se ha evidenciado en otros estudios y propuestas (Flores y Rico, 2015; Murillo et al., 2016; Planas, 2018; Ru-Fen, 2016). No obstante, es importante profundizar en otros estudios el análisis de esta efectividad por cada tipo de problema, sus estructuras y los métodos cognitivos que el estudiante realiza. 
Otra dirección del estudio fue verificar si el programa de gamificación autoconstructiva desarrollaba el aprendizaje de la adición y sustracción en estudiantes de primer grado de primaria en un método preexperimental, de lo cual se obtuvieron resultados favorables al programa. Los resultados en este tipo de aprendizaje fueron significativos $(Z=-5,833 ; p<.01)$, y se evidenció sus beneficios al reducirse la cantidad de niños y niñas en un $80.6 \%$ ubicados en el nivel de inicio al $2.8 \%$, y el incremento de 0 a $77.8 \%$ de sujetos en el nivel de logro. Estos resultados son coherentes con lo hallado en investigaciones que introdujeron la lúdica en los procesos de enseñanza del profesor ante el aprendizaje de las matemáticas (Carlson et al., 2017; Çelik, 2017; Wong, 2018). En cierta medida, aunque no es del todo determinante, la efectividad del programa ha establecido que el juego autónomo en las matemáticas desarrolla el aprendizaje de autoconstrucción en los niños y niñas gracias a que predispone el proceso atencional del estudiante ante la tarea operativa (Carlson et al., 2017; Groos, 1976; Gross, en Martínez, 2008), y se genera mejor predisposición para el alcance progresivo del resultado de alguna operación.

Como el programa lo ha demostrado, resulta importante para el estudio entender que en el pensamiento del estudiante se ejecutan distintos procesos de operación, con distintos modelos de ejecución a la par, lo cual es un factor importante para que el estudiante alcance resultados con menor tiempo, y con más posibilidades de crear tipos de respuesta asertivos con la operación. Gran número de estudiantes antes de llegar al tercer o cuarto grado de primaria (ciclo IV), necesitan establecer herramientas cognitivas de corrección como un medio de metaoperacionalidad, esto deviene de la posibilidad de lograr la modificación de estructuras cognitivas o flexibilizarlas en el estudiante como un operador y constante supervisor de sus mecanismos de operacionalidad (Feurstein en Alpízar, 2016); ello ha ocurrido en algunos estudiantes que lograron desarrollar operaciones bajo representaciones icónicas $(d(+)=34$; e. $=2 ; \mathrm{p}<.01$ ), que es el menor nivel de complejidad en el desarrollo de operaciones matemáticas, lo más difícil para ellos ha sido llegar a la simbolización, y mucho más la algoritmización como en otras propuestas se ha planteado (Lubis \& Nasution, 2017; Ortiz-Colón et al., 2018). Sin embargo, aunque esto no es el propósito de la investigación, es importante señalar que casi un estándar del $45 \%$ del total de sujetos del grupo de experimentación han obtenido nivel de logro en todos los tipos de representación (dimensiones), lo cual es indicativo que en muchos de los estudiantes también se han desarrollado los procesos de representación simbólica y algorítmica, pero con mejor predisposición para la representación icónica de adición y sustracción (Carrillo et al., 2016; Cañellas y Rassetto, 2013). Estos resultados indican que es importante el logro de 
operaciones más complejas en estudiantes de 6 a 8 años de edad, solo si su sistema de desarrollo cognitivo lo permite en dicha edad.

Finalmente, el estudio ha contribuido en descubrir que las técnicas etnoculturales implementadas por recursos propios del contexto andino, en este caso con temática incaica, han permitido que los problemas abordados por los estudiantes del grupo experimental se desarrollasen con mayor efectividad gracias a los rasgos culturales desarrollados en los recursos didácticos, ya que estos desarrollaron mejor interacción entre el profesor y el alumno, y mejor interacción entre el estudiante y el problema, y los efectos etnoculturales de las técnicas aplicadas redujeron la recarga cognitiva en la búsqueda de soluciones gracias al componente lúdico de cada técnica y recurso aplicado; esto pone de manifiesto que la técnicas etnoculturales desde el proceso de gamificación es efectivo para aprendizajes rígidos, verticales o complejos.

En cuanto a la gamificación autoconstructiva, el principal aporte en el aprendizaje de la adición y sustracción es que los componentes lúdicos y autonomía generada en el estudiante, permiten que otros factores como la felicidad, predisposición atencional y motivaciones intrínsecas hayan influenciado en la búsqueda de respuestas a operaciones con determinada complejidad. La gamificación autoconstructiva, entonces, se ha comportado como una variable estratégica de amplificación del sentido perceptivo y constructivo en los estudiantes, que brinda libertad en la búsqueda de respuestas a distintos tipos de operación.

\section{Conclusiones}

1. Los efectos de las técnicas etnoculturales han beneficiado la resolución de problemas luego de verificar las diferencias significativas en favor al grupo experimental $(\mathrm{U}=$ 89,000; $Z=-6,862 ; p<.01)$, a comparación del grupo control. Esto se comprobó en el $52.5 \%$ del total de sujetos del grupo experimental a comparación del $2.5 \%$ del grupo control.

2. Las diferencias en los resultados de la resolución de los cuatro tipos de problemas entre las mediciones pretest y postest: cambio $(\mathrm{U}=230,500 ; \mathrm{p}<.01)$, combinación $(\mathrm{U}=$ 222,500; p <.01), comparación $(U=357,500 ; p<.01)$, igualación $(U=336,500 ; p<.01)$, revelaron la efectividad de las técnicas etnoculturales luego de aplicar el programa de experimentación.

3. El programa de gamificación autoconstructiva también fue efectivo en el aprendizaje de adición y sustracción de 36 sujetos de experimentación, luego de comparar los resultados de las evaluaciones pretest y postest $(Z=-5,833 ; \mathrm{p}<.01)$. Esto se determinó 
en el incremento del 0 al $77.8 \%$ de estudiantes que presentaron nivel de logro luego de la aplicación de dicho programa.

4. La gamificación autoconstructiva también logró beneficios en los tipos de representación del aprendizaje de adición y sustracción con significancia menor al $1 \%$ de probabilidad (icónica: $\mathrm{d}(+)=34 ; \mathrm{p}<.01$; simbólica: $\mathrm{d}(+)=33 ; \mathrm{p}<.01$; algorítmica: $\mathrm{d}(+)=34 ; \mathrm{p}<.01)$.

5. La eficiencia del programa de gamificación autoconstructiva han permitido deducir que la mejora del aprendizaje de adición y sustracción se ha expuesto en los tres tipos de representación con un promediado de aproximadamente el $50 \%$ de sujetos ubicados en el nivel de logro (r. icónica $=38.9 \%$; r. simbólica $=44.4 \%$; r. algorítmica $=47.2 \%$ ). Estos estudiantes lograron efectivizar sus capacidades para agrupar elementos a un conjunto o sustraerlos, asignar elementos gráficos a cierto grupo de elementos, y descomponer o secuencializarlos de acuerdo a cantidades específicas.

\section{Referencias}

Alpízar, L. (2016). La modificabilidad estructural cognitiva en la familia de un enfermo alcohólico. Drugs and Addictive Behavior, 3(2), 283-301. Recuperado de http://www.funlam.edu.co/revistas/index.php/DAB/article/view/2875

Ayal, C.; Kusuma, Y.; Sabandar, J. \& Afgan, J. (2016). The Enhancement of Mathematical Reasoning Ability of Junior High School Students by Applying Mind Mapping Strategy. Journal of Education and Practice, 7(25), 50-58. Recuperado de https://iiste.org/Journals/index.php/JEP/article/view/33035/33931

Bragg, L. A. \& Herbert, S. (2017). A "True" Story about Mathematical Reasoning Made Easy. Australian Primary Mathematics Classroom, 22 (4), 3-6. Recuperado de: https://eric.ed.gov/?q=+logical+reasoning+for+children\&id=EJ1163819

Calsa, G.C. y Furtuoso, P. (2015). Estudo sobre a prática de alfabetização matemática de professoras da educação infantil, Revista Educação e Linguagens, Campo Mourão, 4(6), 124-141. Disponible en:

http://www.fecilcam.br/revista/index.php/educacaoelinguagens/article/view/804

Cánovas, D. (2016). La construcción del concepto de número en el niño durante la etapa de Educación Infantil (Trabajo de fin de grado), Universitat d'Alacant, Alicante, España. Recuperado de: https://rua.ua.es/dspace/handle/10045/56069

Cañellas, A. y Rassetto, M. (2013). Representaciones infantiles sobre las notaciones numéricas. Tecné Episteme y Didaxis TED, 33, 87 - 101. Recuperado de http://revistas.pedagogica.edu.co/index.php/TED/article/view/2035 
Carlson, J., Harris, H. y Harris, K. (2017). Contador de monedas: Gamificación para la gestión del aula. Information Systems Education Journal (ISEDJ). 15 (5), 1 - 11. Recuperado de http://isedj.org/2017-15/n5/ISEDJv15n5p4.html

Carrillo, J.; Contreras, L.C.; Climent, N.; Montes, M.A.; Escudero, D.I. y Flores, E. (2016). Didáctica de las matemáticas para maestros de educación primaria. Ediciones Paraninfo: Madrid.

Çelik, M. (2017). Examination of Children Decision Making Using Clues during the Logical Reasoning Process. Educational Research and Reviews, 12(16), 783-788. Doi https://doi.org/10.5897/ERR2017.3297

D'Amore B. (2006). Didattica della matematica "C". In: Sbaragli S. (ed.) (2006). La matematica e la sua didattica, vent'anni di impegno. Atti del Convegno Internazionale omonimo, Castel San Pietro Terme (Bo), 23 settembre 2006. Bologna: Pitagora. 93-96.

D’Amore, B. e Fandiño, M.I. (2013). La didattica della didattica della matematica: esperienze personali e spunti critici di discussione e ricerca. L'insegnamento della matematica e delle scienze integrate, 36(4), 325 - 353. Recuperado de: http://www.dm.unibo.it/rsddm/it/articoli/damore/807\%20DAmore\%20Fandino\%20Pin illa.pdf

D’Amore, B. e Fandiño, M.I. (2006). Che problema i problemi! L'insegnamento della matematica e delle scienze integrate. 6 (29 AB.), 645 - 664. Recuperado de: http://www.digitaldocet.it/allegati/damore/problemi/588_Problemi.pdf

D’Amore, B.; Fandiño, M.I. e Marazzani, I. (2004). "Esercizi anticipati” e "zona di sviluppo prossimale": comportamento strategico e linguaggio comunicativo in attività di problema solving. La matematica e la sua didattica. 2, 71-95. Recuperado de: http://www.dm.unibo.it/rsddm/it/articoli/damore/481\%20Esercizi\%20anticipati.pdf

Flores, P. y Rico, L. (2015). Enseñanza y aprendizaje de las matemáticas en educación primaria. Madrid, España: Pirámide.

Gross, K. (1976). The Play of Man: Teasing and Love-Play. In: J.S. Bruner; A. Jolly \& K. Sylva (eds.). Play. Its role in development and evolution. Penguin Books Ltd. - International psychoterapy institute: United States of America, $105-131$.

Lee, C. (2016). An Appropriate Prompts System Based on the Polya Method for Mathematical Problem-Solving. Eurasia Journal of Mathematics Science and Technology Education, 13(3), 893-910. doi: https://doi.org/10.12973/eurasia.2017.00649a

Lozano, M. D. (2017). Investigating task design, classroom culture and mathematics learning: an enactivist approach. ZDM Mathematics Education, 49 (6), 895-907. doi: http://dx.doi.org/10.1007/s11858-017-0890-4

Lubis, A. \& Nasution, A. A. (2017). How Do Higher-Education Students Use Their Initial Understanding to Deal with Contextual Logic-Based Problems in Discrete Mathematics?. International Education Studies, 10(5), 72-86. doi: https://doi.org/10.5539/ies.v10n5p72 
Mañeru, G. (2015). Fundamentos pedagógicos de la simulación educativa en el área sanitaria: Competencias docentes. Ediciones Eunate: Madrid, España.

Meloni, C.; Fanari, R.; Bertucci, A. \& Berretti, S. (2017), Impact of Early Numeracy Training on Kindergarteners from Middle-Income Families, 14th International Conference on Cognition and Exploratory Learning in Digital Age (CELDA 2017), University of Cagliari, Cagliari, Italy, https://goo.gl/6BrCMF

Murillo, F., Román, M., y Atrio, S. (2016). Los Recursos Didácticos de Matemáticas en las Aulas de Educación Primaria en América Latina: Disponibilidad e Incidencia en el Aprendizaje de los Estudiantes. Archivos analíticos de políticas educative, 24(67), 2 21. Recuperado de: http://www.redalyc.org/articulo.oa?id=275043450067

Ortiz-Colón, A.-M.; Jordán, J. \& Agredal, M. (2018). Gamificación en educación: una panorámica sobre el estado de la cuestión. Educ. Pesqui., São Paulo, 44 (e173773), 117. DOI: http://dx.doi.org/10.1590/S1678-4634201844173773

Planas, N. (2018). Language as resource: a key notion for understanding the complexity of mathematics learning. Educ Stud Math, 98 (3), 215-229. Doi: https://doi.org/10.1007/s10649-018-9810-y

Rodríguez, E. (2008). El juego como escuela de vida: Karl Groos. Magíster. Revista Miscelánea de Investigación, 22, $7 \quad-\quad 22 . \quad$ Recuperado de: https://dialnet.unirioja.es/servlet/articulo?codigo $=2774872$

Ru-Fen, Y. (2016). Creating Learning Environments for Indigenous Students through Culturedbased Math Modules. Universal Journal of Educational Research, 4(8), 1809-1814. Recuperado de https://files.eric.ed.gov/fulltext/EJ1110800.pdf

Segovia, I. y Rico, L. (2017). Matemáticas para maestros de educación primaria. Madrid, España: Pirámide.

Siegler, R.S. \& Braithwaite, D.W. (2016). Numerical development. Annual Review of Psychology, (Anticipated https://files.eric.ed.gov/fulltext/ED565890.pdf

Schneider, N. C. (2015). Aprendendo e Ensinando Através do Uso de Materiais Recicláveis na Educação Infantil, Revista Monografias Ambientais - REMOA / UFSM, 14, 208 - 214. Disponible en: https://periodicos.ufsm.br/index.php/remoa/article/view/18752

Sungwoong, L. (2016). The Effects of Representation Format in Problem Representation on Qualitative Understanding and Quantitative Proficiency in a Learning Game Context. ProQuest LLC, Ph.D. Dissertation, The Florida State University. Recuperado de https://search.proquest.com/docview/1806862355

Taddia, F. e D’Amore, B. (2015). Perché diamo I numeri?. E tante altre domande sulla matematica. Firenze, Italia: Scienza. 
Tafarelo, A. y Bonanno, A. (2016). A construção do conceito de número e suas implicações na aprendizagem das operações matemáticas, XII Encontro Nacional de Educação Matemática, Educação Matemática na Contemporaneidade: desafios e posibilidades, 1 - 12. Disponible en: http://www.sbem.com.br/enem2016/anais/pdf/5122_3136_ID.pdf

Viterbori, P.; Traverso, L. \& Usai, C. (2017). The Role of Executive Function in Arithmetic Problem-Solving Processes: A Study of Third Graders. Journal of Cognition and Development, 18(5), 595-616. doi: http://dx.doi.org/10.1080/15248372.2017.1392307

Wibowo,T; Sutawidjaja, A.; Rahman As'ari', A. \& Sulandra, I. M. (2017).The Stages of Student Mathematical Imagination in Solving Mathematical Problems. International Education Studies, 10(7), 48-58. doi: https://doi.org/10.5539/ies.v10n7p48

Wong, T. T.-Y. (2018). Is Conditional Reasoning Related to Mathematical Problem Solving?. Developmental Science, 21(5) e12644. Doi: http://dx.doi.org/10.1111/desc.12644

Yorulmaz, A. y Onal, H. (2017). Examen de las opiniones de los maestros de clase sobre los errores que cometen los estudiantes de primaria en cuatro operaciones. Universal Journal of Educational Research. 5 (11), 1885-1895. Recuperado de http://www.hrpub.org/journals/article_info.php?aid=6452 